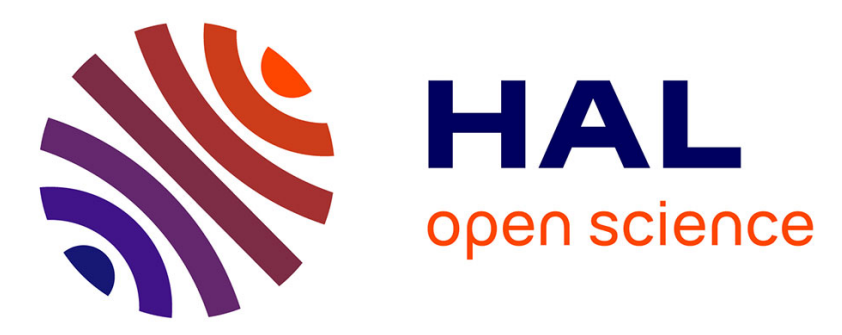

\title{
Investigation of tokamak turbulent avalanches using wave-kinetic formulation in toroidal geometry
}

Camille Gillot, Guilhem Dif-Pradalier, Xavier Garbet, Olivier Panico, Yanick Sarazin, Robin Varennes, David Zarzoso

\section{- To cite this version:}

Camille Gillot, Guilhem Dif-Pradalier, Xavier Garbet, Olivier Panico, Yanick Sarazin, et al.. Investigation of tokamak turbulent avalanches using wave-kinetic formulation in toroidal geometry. Journal of Plasma Physics, 2021. hal-03099961

\section{HAL Id: hal-03099961 https://hal.science/hal-03099961}

Submitted on 6 Jan 2021

HAL is a multi-disciplinary open access archive for the deposit and dissemination of scientific research documents, whether they are published or not. The documents may come from teaching and research institutions in France or abroad, or from public or private research centers.
L'archive ouverte pluridisciplinaire HAL, est destinée au dépôt et à la diffusion de documents scientifiques de niveau recherche, publiés ou non, émanant des établissements d'enseignement et de recherche français ou étrangers, des laboratoires publics ou privés. 


\title{
Investigation of tokamak turbulent avalanches using wave-kinetic formulation in toroidal geometry
}

\author{
Camille Gillot ${ }^{1,2} \dagger$, Guilhem Dif-Pradalier ${ }^{1} \ddagger$, Xavier Garbet ${ }^{1}$, Olivier \\ Panico $^{1}$, Yanick Sarazin ${ }^{1}$, Robin Varennes ${ }^{1}$, David Zarzoso ${ }^{3}$ \\ ${ }^{1}$ CEA, IRFM, F-13108 Saint-Paul-lez-Durance, France \\ ${ }^{2}$ Ecole des Ponts ParisTech, Champs sur Marne, France \\ ${ }^{3}$ Aix Marseille Université, CNRS, PIIM, UMR 7345, Marseille, France
}

(Received $\mathrm{xx}$; revised $\mathrm{xx}$; accepted $\mathrm{xx}$ )

The interplay between toroidal drift wave turbulence and tokamak profiles is investigated using a wave-kinetic description. The coupled system is used to investigate the interplay between marginally stable toroidal drift-wave turbulence and geodesic-acoustic modes (GAM). The coupled system is found to be unstable. Notably, the most unstable mode corresponds to the resonance between the turbulent wave radial group velocity and the GAM phase velocity. For a low-field-side ballooned drift wave growth, a background flow shear breaks the symmetry between inwards- and outwards-travelling instabilities. This mechanism is generic and displays many of the features expected for avalanches in developed tokamak turbulence.

\section{Introduction}

The estimation of the quasi-linear fluxes requires proper knowledge of the turbulent spectrum. This is a complex endeavour. The turbulent fluxes require computing the two-point correlation function $\mathcal{C}_{\phi}\left(t_{1}, t_{2}, \vec{x}_{1}, \vec{x}_{2}\right)$ of the potential (Adam et al. 1979):

$$
\mathcal{C}_{\phi}\left(t_{1}, t_{2}, \vec{x}_{1}, \vec{x}_{2}\right)=\left\langle\phi\left(t_{1}, \vec{x}_{1}\right) \phi\left(t_{2}, \vec{x}_{2}\right)\right\rangle_{\text {turb }}
$$

This correlation function is then weighted appropriately to express the flux carried at $\left(t_{1}, \vec{x}_{1}\right)$ by a plasma parcel that was displaced from $\left(t_{2}, \vec{x}_{2}\right)$. The average $\langle\cdot\rangle_{\text {turb }}$ is taken on realisations of the system and on unobserved symmetry directions. Its determining equations are very complex and can be unpractical for both analytical and numerical works (Farrell \& Ioannou 2007, Srinivasan \& Young 2012). In the case of tokamak plasmas, turbulence is populated by drift-wave-like micro-instabilities at high toroidal mode number, driven by kinetic interchange coupling.

Explanations for turbulent saturation often revolve around mode coupling. Non-linear coupling pours an excess of energy from a turbulent eigenmode to stabler eigenmodes, through modulation by a low wavenumber mode or through scattering with another turbulent modes. In the near-marginal regime, avalanches provide an effective vector for heat transport (Diamond \& Hahm 1995; Newman et al. 1996): bursts travel through the plasma in an almost ballistic fashion (Sarazin \& Ghendrih 1998, Sarazin et al. 2010). Such avalanches have been linked to the transition from Bohm to gyroBohm scaling for turbulent transport (Carreras et al. 1996, Garbet et al. 1999, Lin et al. 2002, Candy \& 
Waltz 2003). They are routinely observed in both gradient-driven and flux-driven nonlinear simulations (Beyer et al. $\mid$ 2000, Idomura et al. 2009, McMillan et al. 2009 , Görler et al. 2011; Dif-Pradalier et al. 2017), although the comparison between gradient-driven and flux-driven dynamics is still an open problem (Rath et al. 2016). The zonal flow patterns found in those simulations tend to be asymmetric, with sometimes strong radial localisation (McMillan et al.|2011, Dif-Pradalier et al. 2015, Zhu et al. 2018a, Ivanov et al. 2020), prompting the nick-name "zonal flow staircase". Several explainations have been advanced for such avalanches, from self-organised criticality in sub-marginal regime (Bak et al. 1987, Hwa \& Kardar 1992; Schekochihin et al. 2012; van Wyk et al. 2016; Pringle et al. 2017; McMillan et al. 2018), to non-linear motion of turbulent potential filaments (Beyer et al. |2000, Sarazin et al. 2010, Gillot 2020). The coupling to the axisymmetric modes and especially zonal flows is of particular interest, because they shear turbulent eddies and act as an efficient mean to saturate turbulence. One of the drivers of the growth of zonal flows is the so-called zonostrophic instability. This instability is due to a modulational coupling of two drift waves giving energy to the sheared zonal flow (Champeaux \& Diamond 2001; Diamond et al. 2005). When turbulent structures and zonal flows act on different radial and temporal scales, individual modulations can be thought of as infinitesimal: the problem can be modelled through the dynamics of a turbulent spectrum, alone forgetting individual wave-wave interactions. This approach has been applied to the modelling of drift waves, on temperature gradient and trapped electron modes, see Anderson et al. $(2002,2006)$; Srinivasan \& Young (2012); Parker (2016); Gillot (2016); Ruiz (2017); Zhu et al. (2018b).

Top-of-the-shelf quasi-linear models often rely on quasi-stationary ansatz to close the turbulent spectrum (Citrin et al. 2017). This choice has been criticized as being unable to account for turbulent self-organisation features like zonal flow growth and avalanching for near-marginal turbulence. Wave-kinetic modelling (Weinberg 1962) attempts to estimate the fluctuation spectrum in a simplified manner. The two-point correlations decays as the scale of the turbulent structures. If turbulent structures are much smaller than the background profile scale, the two-point separation can be represented in Fourier space as a local turbulent spectrum $\mathcal{I}(\vec{x}, \vec{k})$. As a result, both the driving gradients and the zonal flows are assumed axisymmetric and radially smooth. They should evolve slowly enough for the turbulent structures to adapt adiabatically. In these conditions, an eikonal approach is accessible. By neglecting non-linear saturation mechanisms, the dynamics can be reduced to a kinetic equation on the spectrum $\mathcal{I}$. The so-called wave-kinetic equation is written as

$$
\partial_{t} \mathcal{I}+\partial_{\vec{k}} \omega \cdot \partial_{\vec{x}} \mathcal{I}-\partial_{\vec{x}} \omega \cdot \partial_{\vec{k}} \mathcal{I}=2 \gamma \mathcal{I}+\text { Saturation }
$$

The saturation term corresponds to non-linear couplings between turbulent cells, and the saturation level they prescribe. In full generality, this term requires higher-order correlation functions. By analogy with the Boltzmann equation, it is often approximated by some kind "eddy-eddy" collision operator (Ruiz et al. 2019). Here, $\omega+i \gamma$ is the eigenmode angular frequency and growth rate, computed from the dispersion relation. The variable $\mathcal{I}$ represents a conserved wave action. It can be defined using the Wigner density function of the potential, or equivalently the Fourier-transform of the two-point correlation function $\mathcal{C}_{\phi}$ :

$$
\left.\mathcal{I}(t, \vec{x}, \vec{k}) \delta(\omega-\omega(t, \vec{x}, \vec{k}))=\partial_{c} \mathcal{D} \int \mathcal{C}_{\phi}\left(t+\frac{\tau}{2}, t-\frac{\tau}{2}, \vec{x}+\frac{\vec{s}}{2}, \vec{x}-\frac{\vec{s}}{2}\right) \mathrm{e}^{i \omega \tau-i \vec{k} \cdot \vec{s}} \mathrm{~d} \vec{s} \mathrm{~d} t 1.3\right)
$$

where the function $\partial_{c} \mathcal{D}$ will be made explicit later. The $\delta$ function relies on a strong time scale separation between the wave frequency and the evolution of background profiles. 
The choice of a wave-kinetic formulation in opposition to an eikonal formulation is not without consequences. The non-linear coupled evolution of the amplitude $\mathcal{A}_{p}(t, \vec{x})$ and phase $\sigma_{p}(t, \vec{x})$ of individual wave packets $\phi_{p}=\mathcal{A}_{p}(t, \vec{x}) \exp i \sigma_{p}(t, \vec{x})$ is lost, and replaced by a statistical description. One may expect to lose valuable phase information, and the associated phase dynamics. Nevertheless, the wave-kinetic approach has been successfully implemented for the drift wave coupling to zonal flows in Parker (2015, 2016); Gillot (2016); Ruiz et al. (2016); Ruiz (2017); Zhu et al. (2018b).

In this framework, and in a simplified slab geometry, a seed zonal flow shears the turbulent eddies, making the spectrum $\mathcal{I}$ asymmetric in $k_{r}$. This induces a non-zero Reynolds stress $\mathcal{R}$, which reinforces the zonal flow:

$$
\mathcal{R}(t, \vec{x})=\int \frac{k_{r} k_{\theta}}{\mathcal{D}_{c}} \mathcal{I}(t, \vec{x}, \vec{k}) \frac{\mathrm{d} \vec{k}}{(2 \pi)^{2}}
$$

As a consequence, the zonal flow grows as $\gamma \propto k_{r, \mathrm{ZF}} \sqrt{\mathcal{E}}$ with $\mathcal{E}$ the turbulent energy, proportional to $\mathcal{I}$. This growth rate diverges at high zonal flow wavenumber $k_{r, \mathrm{ZF}}$. Actually, for thinner zonal flows, the free-energy source that is the density gradient is modified. The associated diamagnetic drift is sheared in the opposite direction $(b \times \nabla n \sim$ $\left.-k_{r, \mathrm{ZF}}^{2} u_{E}\right)$. The zonal flow growth is stabilised (Parker 2016), with a weaker global growth as $\gamma \propto k_{r, \mathrm{ZF}} \sqrt{\mathcal{E}} \sqrt{1-k_{r, \mathrm{ZF}}^{2} / k_{\perp, \mathrm{turb}}^{2}}$.

In the case of a tokamak plasma, toroidicity makes everything more complicated. On the one hand, turbulence has to make do with ballooning and magnetic shear. The radial mode number results from a competition between polarisation, magnetic shear and parallel acoustic dynamics. This severely impacts the shearing effect on turbulence (Garbet et al. 2002) by providing an effective recall towards low-field-side ballooning. This constrains the accessible transverse mode numbers. On the other hand, the response of the zonal flows is also modified, as it is coupled to the Landau-damped geodesic acoustic modes (GAM) Qiu et al. 2018.

GAMs have been shown to have a mitigating effect on turbulence by various authors (Hallatschek \& Biskamp 2001; Waltz \& Holland 2008). In addition, simulations with both ITG and energetic-particle-driven GAMs (EGAMs) feature increased turbulent avalanches, synchronised at the EGAM frequency (Zarzoso et al. 2013). Furthermore, the non-linear interaction of a GAM on an ITG mode can produce another ITG mode through parametric decay (Girardo 2015). ITG turbulence has a radial group velocity which scales with the magnetic drift. In certain conditions, the slab zonostrophic instability has been shown to have a travelling branch (Ruiz et al. 2016). GAMs have a radial phase velocity that the slab Euler equation does not have, which also scales as the magnetic drift velocity. When the radial motion of turbulent structures matches the GAM's, the turbulent wave gets trapped inside the GAM (Sasaki 2018 ; Sasaki et al. $2018 b)$. The coupled system is unstable and features travelling solutions (Sasaki et al.|2016, 2018a). These unstable solutions are investigated as candidates for turbulent avalanches.

The wave-kinetic equation is among the simplest models for the evolution of the turbulent spectrum used by the quasi-linear model. We investigate some of the added features of this extended quasi-linear model in relation. In particular, we formulate the coupling between an axisymmetric gyro-kinetic description and a wave-kinetic description of the turbulent spectrum, and interrogate whether this model is able to recover some self-organisation features. In particular, we propose to extend the model from Sasaki et al. (2018a) to the kinetic description of GAMs. Along the way, we derive (section 2 ) a self-consistent wave-kinetic equation for any dispersion relation, along with its backreaction on the profiles (section 3 . As a consistency check, we apply the formalism to 
a simple slab drift-wave model (section 4). We model the laminar profiles using the axisymmetric component of the Vlasov equation, keeping the poloidal dependency to access GAM dynamics. The non-axisymmetric components are modelled using the wavekinetic equation, using a general dispersion relation for the ITG mode (section 5 and 6 .

We show that the GAM radial phase velocity and the wave-kinetic radial advection resonate, destabilising the GAM mode into a radially moving zonostrophic instability. When introducing a population of energetic particles, this resonance happens at the EGAM frequency, and so does the zonostrophic instability. When the turbulent growth rate is poloidally uniform, neither an up-down asymmetry in the turbulent intensity nor a background flow shear are enough to introduce a preferred radial direction for the unstable mode. However, this asymmetry can be triggered by the cooperation between both a background flow shear and a turbulent growth ballooned on the low-field side. The direction of this asymmetry is consistent with the observation from Idomura et al. (2009); McMillan et al. (2009): avalanches propagate according to the sign of the background zonal flow shear rate. These features make this travelling unstable coupled mode between turbulence and GAMs a candidate explanation for turbulent avalanche processes.

\section{Derivation of the wave-kinetic equation}

The wave-kinetic equation models small-scale waves as pseudo-particles inside the plasma. The waves should maintain their coherence at their scale, and should only be affected by local properties of the background plasma. The pseudo-particles move according to geometrical optics. Their spatial motion is given by their group velocities. In an inhomogeneous or dispersive medium, the waves are distorted, and their wavenumber change.

The wave-kinetic equation has found various applications in plasma physics since its introduction by Weinberg (1962). Its use in turbulence modelling has often relied on adhoc formulations like in Diamond et al. (2005); Sasaki et al. (2018a). Conversely, several authors have attempted a simple self-consistent formulation of this model (Dodin \& Fisch 2012, Parker 2016, Ruiz 2017), and found earlier versions to be missing essential physics for the saturation of the zonal flows (Parker 2015, Ruiz et al. 2016).

We consider in the following a bath of ideal waves, oscillating at high frequency and high wavenumber, in an inhomogeneous medium with slower and smoother evolution. As an extension to the quasi-linear framework, the wave-kinetic equation relies on the same assumptions as the usual quasi-linear computations of the matter and heat fluxes: the temporal scale separation between the wave dynamics and the profiles' dynamics, and a spatial scale separation between the local description of the ITG mode and of the smoother profiles and zonal flows. In our case, the fastest time scale for the dynamics of the profiles is the GAM, corresponding to the assumption $\omega_{\text {turb }} \gg \omega_{G A M}$. The scale separation allows to treat the waves as point particles, neglecting their finite correlation length, and their response to profile evolution instantaneous. The radial scale separation on the other hand may only be marginal, because of the strong radial pattern of zonal flow staircases (Dif-Pradalier et al. 2015, Ivanov et al. 2020). The case of non-ideal waves is plagued by numerous technical and fundamental difficulties (Brillouin 1960) we avoid here by restricting ourselves to the ideal case. This is possible as long as $\gamma_{t u r b} \ll \omega_{G A M}$. For ITG modes, this is typically the case.

Our derivation follows the one in Whitham (1965); Jimenez \& Whitham (1976); Kaufman et al. (1987), based on a postulated wave action principle. The idea is to define a variational principle for the waves, and to derive the wave-kinetic and Poisson equation 
from it. Using an action principle will allow to self-consistently derive the dynamics when the turbulent background is coupled to the evolution of the profiles, while ensuring the correct conservation properties. In the limit of ideal waves, this derivation is equivalent to the one by McDonald \& Kaufman (1985); McDonald (1988); Dodin \& Fisch (2012), while being algebraically much simpler for resonant instabilities. Local drift-wave modes such as the ITG obey a scalar dispersion $\mathcal{D}(c, r, \zeta)$ in mixed Fourier space for the local ITG modes. $n$ is the toroidal mode number, $c=\omega / n$ the mode angular phase velocity, $r$ the reference radial position of the mode, and $\zeta=k_{r} / n q^{\prime}$ its ballooning angle. By symmetry, we assume $n>0$. The eigenfrequency is obtained as a function of $r$ and $\zeta$ by solving $\mathcal{D}(c)=0$. In case of multiple branches, an index can be introduced to lift the ambiguity. We are neglecting growth and damping of the waves. In the kinetic regime, those are carried by the imaginary part of $\mathcal{D}$. Incidentally, we assume $\mathcal{D}$ to be real.

As a starting point, we recast it as an action principle

$$
\mathcal{S}=\int \frac{\mathcal{N} e^{2}}{2 T} \mathcal{D}(c, r, \zeta, n)|\phi|^{2} r \mathrm{~d} r n^{2} q^{\prime} \mathrm{d} \zeta \mathrm{d} c
$$

where $\mathcal{N}$ is the plasma density, $T$ its temperature, and $e$ the electron charge. With this formulation, the normalisation of $\mathcal{D}$ has to be chosen with care to ensure the consistency between the first-principle action and this reduced $\mathcal{S}$. See paragraph 4 for an example.

Equivalently, the mode dispersion relation is obtained by setting $\partial \mathcal{S} / \partial|\phi|^{2}=0$. A scalar dispersion relation $\mathcal{D}$ is defined up to a function of $r, \zeta, n$. The normalisation of the integrand is chosen so as to retrieve the Poisson variational principle in the high frequency limit.

$$
\mathcal{S} \underset{c \rightarrow \infty}{\longrightarrow} \int \frac{\mathcal{N} e^{2}}{2 T} k_{\perp}^{2} \rho_{i}^{2}|\phi|^{2} r \mathrm{~d} r n^{2} q^{\prime} \mathrm{d} \zeta \mathrm{d} c
$$

The wave-kinetic equation describes the behaviour of the amplitude of turbulent waves and abstracts out their precise shape. This argument can be made precise by introducing an amplitude-phase decomposition of the potential as a sum of wave-packets $p$

$$
\phi(t, r, \theta, \varphi)=\sum_{p} \sqrt{\frac{2 T \mathcal{A}_{p}(t, r)}{\mathcal{N} e^{2}}} \exp \left(i \sigma_{p}(t, r, \theta)+i n \varphi\right)
$$

where $\mathcal{A}_{p}$ plays the role of the energy in the fluctuation, and $\sigma_{p}$ is a real phase function. $\mathcal{A}_{p}$ is a very smooth function, while $\sigma_{p}$ contains the fine details. To avoid cluttering the notation, the subscript $p$ will remain implicit except when otherwise noted.

The computations leading to 2.1 can be re-done using $\omega=-\partial_{t} \sigma, k_{r}=\partial_{r} \sigma$ instead of the ballooning phase function $\sigma=-n c t-n q(\theta-\zeta)$, and neglecting the second derivatives of $\sigma$. These second derivatives are related to the coherence and finite extent of the waves, and are neglected by construction. $\mathcal{A}$ is assumed to be constant at the scale of the waves, so its derivatives are neglected. This allows to define the eikonal action principle

$$
\mathcal{S}_{\text {eik }}=\sum_{p} \int \mathcal{D}\left(t, c=-\frac{\partial_{t} \sigma_{p}}{n}, r, \zeta=\frac{\partial_{r} \sigma_{p}}{n q^{\prime}}, n\right) \mathcal{A}_{p}(t, r) r \mathrm{~d} r \mathrm{~d} t
$$

with the exact same dispersion relation $\mathcal{D}$. The variations of $\mathcal{S}_{\text {eik }}$ with respect to $\mathcal{A}$ give the dispersion relation 2.5, but applied to derivatives of $\sigma$. The wave conservation 
equation 2.6 corresponds to the variations of $\mathcal{S}_{\text {eik }}$ with respect to $\sigma$.

$$
\begin{array}{r}
\mathcal{D}\left(t, c=-\frac{\partial_{t} \sigma}{n}, r, \zeta=\frac{\partial_{r} \sigma}{n q^{\prime}}, n\right)=0 \\
\partial_{t}\left(\mathcal{A} \partial_{c} \mathcal{D}\right)-\frac{1}{r} \partial_{r}\left(r \mathcal{A} \frac{\partial_{\zeta} \mathcal{D}}{q^{\prime}}\right)=0
\end{array}
$$

Those two equations are valid for each wave packet individually. By analogy with traditional mechanics, 2.5 is called the Hamilton-Jacobi equation. In resolved form, it would write $c\left(x, \partial_{r} \sigma\right)=-\partial_{t} \sigma$ with $c(x, k)$ the wave phase velocity from the dispersion relation. The phase function $\sigma$ serves as a pilot wave. It has a similar role as Hamilton's function for the motion of the individual turbulent waves: it provides the evolution of the canonical momentum $k=\partial_{x} \sigma$ as a function of space and time. Equation 2.6 is already in a conservative form. The convected quantity is $\mathcal{A} \partial_{c} \mathcal{D}$, the ratio of an energy to a toroidal phase velocity. It represents the toroidal momentum stored in the wave packet. We note that the derivative $\partial_{c} \mathcal{D}$ must not vanish. This excludes from this description the case of reactive instabilities, caused by the encounter of two stable branches. Conversely, this description is adequate for kinetic excited or damped waves, for which $\partial_{c} \mathcal{D} \neq 0$. The system 2.5 2.6 is of two non-linear equations, thus unpractical for a bath of wave packets. In order to derive the wave-kinetic equation, we introduce the Wigner density function (Moyal \& Bartlett 1949, McDonald \& Kaufman 1985, McDonald 1988, 1991)

$$
\begin{aligned}
\mathcal{W}(t, r, \zeta) & =\frac{\mathcal{N} e^{2}}{2 T} \int \phi\left(t, r+\frac{x}{2}\right) \phi^{*}\left(t, r-\frac{x}{2}\right) \exp \left(-i n \zeta q^{\prime} x\right) \frac{n q^{\prime} \mathrm{d} x}{2 \pi} \\
& \approx \sum_{p} \mathcal{A}_{p} \delta\left(\zeta-\frac{\partial_{r} \sigma_{p}}{n q^{\prime}}\right)
\end{aligned}
$$

where $\delta$ is the Dirac distribution. The second equality is valid thanks to the radial scale separation between $\mathcal{A}$ and $\sigma$. The function $\mathcal{W}$ encodes both the amplitude and the phase. It serves as a Klimontovitch distribution for the wave packets. Using Whitham's equation 2.6. the convection of the wave action density $\mathcal{W}$ can be computed as

$$
\begin{aligned}
\partial_{t}\left(\mathcal{W} \partial_{c} \mathcal{D}\right)-\frac{1}{r} \partial_{r}\left(r \mathcal{W} \frac{\partial_{\zeta} \mathcal{D}}{q^{\prime}}\right) & =-\left[\partial_{c} \mathcal{D} \frac{\partial_{t r}^{2} \sigma}{n}-\partial_{\zeta} \mathcal{D} \partial_{r}\left(\frac{\partial_{r} \sigma}{n q^{\prime}}\right)\right] \partial_{\zeta} \mathcal{W} \\
& -2 \mathcal{W}\left[\partial_{c} \mathcal{D} \partial_{t} \Im \sigma+\frac{\partial_{\zeta} \mathcal{D}}{q^{\prime}} \partial_{r} \Im \sigma\right]
\end{aligned}
$$

The first square bracket quantifies how radially neighbouring wave trajectories get pulled apart. It will yield the wave stretching term in the wave-kinetic equation. The second square bracket contains the growth rate $\gamma=-\partial_{t} \Im \sigma$ computed using the dispersion relation. As we assume ideal waves, $\gamma=0$. Finally, $\partial_{r} \sigma$ is real by assumption, corresponding to propagating waves (Suchy 1981). To compute the first square bracket, we differentiate the Hamilton-Jacobi equation 2.5 with respect to $r$

$$
\begin{gathered}
0=\partial_{r}\left[\mathcal{D}\left(c=-\frac{\partial_{t} \sigma}{n}, r, \zeta=\frac{\partial_{r} \sigma}{n q^{\prime}}, n\right)\right]=-\partial_{c} \mathcal{D} \frac{\partial_{t r}^{2} \sigma}{n}+\partial_{\zeta} \mathcal{D} \partial_{r}\left(\frac{\partial_{r} \sigma}{n q^{\prime}}\right)+\partial_{r} \mathcal{D} \\
\partial_{t}\left(\mathcal{W} \partial_{c} \mathcal{D}\right)-\frac{1}{r} \partial_{r}\left(r \mathcal{W} \frac{\partial_{\zeta} \mathcal{D}}{q^{\prime}}\right)=-\frac{\partial_{r} \mathcal{D}}{q^{\prime}} \partial_{\zeta} \mathcal{W}+2 \gamma \partial_{c} \mathcal{D} \mathcal{W}
\end{gathered}
$$

The parameters to $\mathcal{D}$ in equation 2.10 are still the derivatives of $\sigma$. In order to replace mentions of $\partial_{r} \sigma$ by $\zeta$, we use the absorbing property of the Dirac distribution $f$ inside $\mathcal{W}$.

$\dagger$ This replacement is only possible on functions $f$ that directly "touch" the $\delta$ distribution: 
We get the wave-kinetic equation as

$$
\partial_{t}\left(\mathcal{W} \partial_{c} \mathcal{D}\right)-\frac{1}{r} \partial_{r}\left(r \mathcal{W} \frac{\partial_{\zeta} \mathcal{D}}{q^{\prime}}\right)+\partial_{\zeta}\left(\mathcal{W} \frac{\partial_{r} \mathcal{D}}{q^{\prime}}\right)=2 \gamma \partial_{c} \mathcal{D} \mathcal{W}
$$

The value of $\partial_{t} \sigma$ is completely defined by the Hamilton-Jacobi equation 2.5 as a function of $r$ and $\zeta . \sigma$ is completely eliminated from the description. The equation on $\mathcal{W}$ can be recast as a conservation for the wave action $\mathcal{I}$

$$
\begin{aligned}
\mathcal{I} & =\mathcal{W} \partial_{c} \mathcal{D}=\frac{\mathcal{N} e^{2}}{2 T}|\phi|^{2} \delta\left(\zeta-\frac{\partial_{r} \Re \sigma}{n q^{\prime}}\right) \partial_{c} \mathcal{D} \\
\partial_{t} \mathcal{I}+\frac{1}{r} \partial_{r}\left(r v_{g}^{r} \mathcal{I}\right)+\partial_{\zeta}\left(v_{g}^{\zeta} \mathcal{I}\right) & =2 \gamma \mathcal{I}
\end{aligned}
$$

The growth rate arises from the solution of the complex analytic dispersion relation 2.5 . The group velocity $v_{g}^{r}$ and the wave distortion $v_{g}^{\zeta}$ given by the usual formulas

$$
\begin{aligned}
q^{\prime} v_{g}^{r} & =-\frac{\partial_{\zeta} \mathcal{D}}{\partial_{c} \mathcal{D}}=\left(\frac{\partial c}{\partial \zeta}\right)_{\mathcal{D}=0} \\
q^{\prime} v_{g}^{\zeta} & =\frac{\partial_{r} \mathcal{D}}{\partial_{c} \mathcal{D}}=-\left(\frac{\partial c}{\partial r}\right)_{\mathcal{D}=0} \\
\gamma & =-n \frac{\Im[\mathcal{D}]}{\partial_{c} \mathcal{D}}
\end{aligned}
$$

Equation 2.13 is a kinetic equation. The waves are advected in phase space so as to conserve the wave angular phase velocity $c(r, \zeta) \cdot c(r, \zeta)$ actually serves as a Hamiltonian for the waves. The additional $n$ factor in the growth rate 2.16 comes from the choice of the phase velocity $c$ as a variable of interest, instead of the frequency $\omega$. The turbulent energy can be derived using Noether's theorem from the eikonal action 3.10, and coincides with the usual definition in dispersive media (Landau \& Lifschitz 1984, eq. 61.9)

$$
E_{\text {turb }}=\int c \mathcal{I} \mathrm{d} \zeta r \mathrm{~d} r=\int \partial_{c}[c \mathcal{D}] \frac{\mathcal{N} e^{2}}{T}|\phi|^{2} \mathrm{~d} \zeta r \mathrm{~d} r
$$

where $c$ is the real solution to the real part of the dispersion relation 2.5. By removing the function $\sigma$ from the description, the non-linear phase dynamics associated to the Hamilton-Jacobi equation 2.5 is lost. Only a linearised version is kept, in the form of the advection velocities $v_{g}^{r}$ and $v_{g}^{\zeta}$.

Dimensionally, the total wave action $\int \mathcal{I} \mathrm{d} \zeta$ from 2.12 is an energy divided by a toroidal angular velocity. It represents the momentum of turbulence when waves are sped up toroidally. Because of the parallel alignment of the turbulent structures, $q \mathcal{I}$ serves as a poloidal momentum.

The growth rate is not self-consistent. This is expected: this description conserves energy, but does not contain the depletion of the free energy source. In order to obtain a self-consistent system of equations, we need to write the associated energy depletion in the evolution of the profiles. This problem is notoriously subtle. It involves the computation of quasi-linear particle fluxes, as well as introducing a ponderomotive force into the system. For reference, see McDonald et al. (1985); Kaufman et al. (1987). In the following, we will consider marginally stable modes, $\gamma=0$. As a consequence, the quasi-linear energy fluxes will be zero.

$f(a) \delta(a-b)=f(b) \delta(a-b)$. If a derivative is present, the expression needs to be shuffled to get it out of the way: $f(a) \partial_{b} \delta(a-b)=\partial_{b}[f(a) \delta(a-b)]=\partial_{b}[f(b) \delta(a-b)]$. 


\section{Coupling to the profile}

Equation 2.13 is coupled to the Vlasov equation for the axisymmetric component of the distribution function $\mathcal{F}$

$$
\partial_{t} \mathcal{F}+\operatorname{div} \vec{\Gamma}_{\text {traj }}+\operatorname{div} \vec{\Gamma}_{\text {turb }}=\text { Sources }+ \text { Collisions }
$$

$\vec{\Gamma}_{\text {traj }}$ is the flux governed by the advection of $\mathcal{F}$ by the trajectories of the gyrocentres, while $\vec{\Gamma}_{\text {turb }}$ contains the heat flux coming from the non-linear coupling of non-axisymmetric fluctuations. The latter flux has components along directions $r, \theta$ and energy $E$. In our wave-centred description, $\vec{\Gamma}_{\text {turb }}$ is approximated as the quasi-linear flux, an integral over the spectrum $\mathcal{I}$. The integrands encode the efficiency of turbulent transport depending on the class of particles. Those depend on the profiles, their gradients and on the wave phase space. Let the linear response $f$ of the distribution function to the wave $\phi$ be written symbolically as

$$
\frac{f}{\mathcal{F}}=\Lambda\left[c=-\frac{\partial_{t} \sigma}{n}, \zeta=\frac{\partial_{r} \sigma}{n q^{\prime}}, n ; r, \theta, E, \mu\right] \frac{e \phi}{T}
$$

For a drift-kinetic system, the dispersion relation with adiabatic electrons can be written as

$$
\mathcal{D}_{\text {adiab }}=\tau+k_{\perp}^{2} \rho_{i}^{2}-\int \Lambda \frac{\mathcal{F}}{\mathcal{N}} \mathrm{d}^{3} v
$$

with $\tau=T / T_{e}$ the ion to electron temperature ratio, and $k_{\perp}^{2} \rho_{i}^{2}$ corresponds to the ion polarisation. The extension to a gyro-kinetic model is straightforward by inserting the appropriate gyro-averaging. The quasi-linear fluxes of gyro-centres read as follows

$$
\begin{aligned}
\Gamma_{\text {turb }}^{r} & =\left\langle v_{E}^{r *} f\right\rangle_{\text {turb }} \\
& =-\Re \sum_{n>0} \frac{i n q \phi^{*}}{r B} \Lambda \frac{e \phi}{T} \mathcal{F} \\
& =-\frac{2 \mathcal{F}}{e \mathcal{N}} \Re \sum_{n>0} \int \frac{i n q}{r B} \Lambda \frac{\mathcal{I}}{\partial_{c} \mathcal{D}} \mathrm{d} \zeta \\
\Gamma_{\text {turb }}^{\theta} & =\left\langle v_{E}^{\theta *} f\right\rangle_{\text {turb }}=\frac{2 \mathcal{F}}{e \mathcal{N}} \Re \sum_{n>0} \int \frac{i n q^{\prime} \zeta}{B} \Lambda \frac{\mathcal{I}}{\partial_{c} \mathcal{D}} \mathrm{d} \zeta \\
\Gamma_{\text {turb }}^{E} & =-e\left\langle\dot{\vec{X}} \cdot \vec{\nabla} \phi^{*} f\right\rangle_{\text {turb }} \\
& =\frac{2 \mathcal{F}}{e \mathcal{N}} \Re \sum_{n>0} \int i n q\left(v_{D}^{\theta}-\frac{q^{\prime}}{q} \zeta v_{D}^{r}\right) \Lambda \frac{\mathcal{I}}{\partial_{c} \mathcal{D}} \mathrm{d} \zeta
\end{aligned}
$$

where the brackets $\langle\cdot\rangle_{\text {turb }}$ denote a sum over all the turbulent modes for all $n$ and $\zeta$. The turbulent intensity is inserted using its definition equation 2.12 .

It should be noted that the total quasi-linear charge flux $\left(\sum_{s} e_{s} \int \Gamma_{\text {turb }, s}^{r} \mathrm{~d}^{3} v\right)$ vanishes. In the case of adiabatic electrons, the ion particle flux is ambipolar. This can be seen by integrating 3.4 and 3.5 on velocity.

$$
\sum_{s} e_{s} \Gamma_{\text {turb }, s}^{r}=\frac{2}{\mathcal{N}} \sum_{n>0} \int \frac{n q}{r B} \Im\left[\sum_{s} \int \mathcal{F}_{s} \Lambda_{s} \mathrm{~d}^{3} v\right] \frac{\mathcal{I}}{\partial_{c} \mathcal{D}} \mathrm{d} \zeta
$$

The factor inside the brackets is the total density response. The dispersion relation 3.3 tells us it is purely real. Hence, the total charge flux vanishes. This is unexpected: we 
should get a polarisation flux carried by the turbulence. This polarisation flux contains the Reynolds stress responsible for the growth of zonal flows (Taylor 1915).

There is actually no issue with equations 3.4 and 3.5 . The vanishing of the polarisation flux is consistent with our ordering on radial derivatives $\partial_{r} \ll n q^{\prime} \zeta$. Because of this ordering, quantities involving an odd number of derivatives are purely imaginary. Instead, the polarisation flux is frozen in the turbulence, and appears as a additional charge density in the Poisson equation. For consistency, we need to adapt the Poisson equation to the eikonal action principle 2.4 .

The quasi-linear energy flux suffers from a similar fate. The total energy flux can be decomposed into the effect of resonant wave-particle interactions and of the non-resonant ponderomotive effect. We suppose that the modes are marginally stable, so that the dispersion relation is real. As a consequence, there is no direct energy exchange between the particles and the wave, so the resonant energy flux is zero. The ponderomotive energy flux is related to the gradient of the turbulent intensity $\mathcal{I}$. Because of the wave-kinetic ordering on the derivatives, the ponderomotive contribution vanishes. This corresponds to a Boussinesq approximation on the turbulent energy content, akin to what is done with the gyro-kinetic polarisation (Scott \& Smirnov 2010). We are left with no quasi-linear energy flux $\Gamma^{E}=0$.

Let $\Phi$ be the axisymmetric electrostatic potential. This potential is associated to a poloidal zonal flow angular velocity $u_{E}=\partial_{r} \Phi / r B$. Derivations of a dispersion relation are typically done in the toroidally rotating plasma frame, where the zonal flow vanishes. In order to move back to the laboratory frame, we introduce the zonal flow as a toroidal Doppler shift

$$
\mathcal{D}\left(c=-\frac{\partial_{t} \sigma}{n}, r, \zeta=\frac{\partial_{r} \sigma}{n q^{\prime}}, n ; u_{E}\right)=\mathcal{D}\left(c=-\frac{\partial_{t} \sigma}{n}+q u_{E}, r, \zeta=\frac{\partial_{r} \sigma}{n q^{\prime}}, n\right)
$$

The complete action principle becomes

$$
\begin{aligned}
\mathcal{S} & =\int \mathcal{D}\left(c=-\frac{\partial_{t} \sigma}{n}+\frac{q \partial_{r} \Phi}{r B}, r, \zeta=\frac{\partial_{r} \sigma}{n q^{\prime}}, n\right) \mathcal{A}(t, r) r \mathrm{~d} r \\
& +\int \frac{m \mathcal{N}}{2 B^{2}}\left(\vec{\nabla}_{\perp} \Phi\right)^{2} r \mathrm{~d} r+\int \frac{\mathcal{N} e^{2}}{2 T_{e}}\left(\Phi-\Phi_{\mathrm{FS}}\right)^{2} r \mathrm{~d} r-\int e \Phi \mathcal{F} \mathrm{d}^{3} v r \mathrm{~d} r
\end{aligned}
$$

The first line is our eikonal action. The second line contains the kinetic energy stored inside the zonal flow and the potential energy of the particles. As usual, the Poisson equation is obtained from the variations of $\mathcal{S}$ with respect to $\Phi$.

$$
\begin{aligned}
-\operatorname{div}_{\perp}\left(\frac{m \mathcal{N}}{B^{2}} \vec{\nabla}_{\perp} \Phi\right)+\frac{\mathcal{N} e^{2}}{T_{e}}\left(\Phi-\Phi_{\mathrm{FS}}\right)-e \int \mathcal{F} \mathrm{d}^{3} v & =\frac{1}{r} \partial_{r}\left[r \frac{\partial \mathcal{D}}{\partial\left(\partial_{r} \Phi\right)} \mathcal{A}(t, r)\right] \\
& =\operatorname{div}_{r} \int \frac{q \mathcal{I}}{r B} \mathrm{~d} \zeta
\end{aligned}
$$

where the last equality uses 3.9 and the definition of the wave packet density $\mathcal{I}$. Turbulence is affected by the poloidal flow $u_{E}$. Modifying the flow costs energy according to a momentum $q \mathcal{I}$. This momentum is equivalent to a polarisation for the $E \times B$ flow. By redefining the distribution function $\mathcal{F}$, this charge could be added to the Vlasov equation as our dearly missed polarisation flux. The final system of equations is composed of 2.13 , 3.1 and 3.11. Once again, the total energy can be derived using Noether's theorem on 
the action 3.10 (see Brizard \& Tronko 2011, appendix))

$$
\begin{aligned}
E_{\mathrm{tot}} & =\frac{\delta \mathcal{S}}{\delta \Phi} \cdot \Phi+\frac{\partial \mathcal{S}}{\partial\left[\partial_{t} \sigma\right]} \cdot \partial_{t} \sigma-\mathcal{S}=E_{\text {kin }}+E_{\mathrm{pol}}+E_{\mathrm{turb}} \\
E_{\mathrm{kin}} & =\int\left[\frac{m}{2} v_{\|}^{2}+\mu B\right] F \mathrm{~d}^{3} v r \mathrm{~d} r \mathrm{~d} \theta \\
E_{\mathrm{pol}} & =\int \frac{m \mathcal{N}}{2 B^{2}}\left|\vec{\nabla}_{\perp} \Phi\right|^{2} r \mathrm{~d} r \mathrm{~d} \theta+\int \frac{\mathcal{N} e^{2}}{2 T_{e}}\left(\Phi-\Phi_{\mathrm{FS}}\right)^{2} r \mathrm{~d} r \mathrm{~d} \theta \\
E_{\mathrm{turb}} & =\int\left[c+q \frac{\partial_{r} \Phi}{r B}\right] \mathcal{I} \mathrm{d} \zeta r \mathrm{~d} r \mathrm{~d} \theta
\end{aligned}
$$

$E_{\text {kin }}$ is the energy stored in kinetic form by the particles. $E_{\text {pol }}$ is the energy stored in the zonal electric field, as mostly as the kinetic energy of the zonal flow $m \mathcal{N} u_{E}^{2} / 2$. $E_{\text {turb }}$ is the energy stored in the turbulence, essentially in the form of kinetic energy of the turbulent $E \times B$ velocity, eventually screened by the response of the plasma. The Vlasov equation 3.1 is constructed so as to follow the motion of particles according to the Hamiltonian $\frac{m}{2} v_{\|}^{2}+\mu B+e \Phi$. Similarly, the wave-kinetic equation 2.13 is constructed so as to follow $c+q \frac{\partial_{r} \Phi}{r B}$ along the trajectories in phase space. Both dynamics allow one to verify the energy conservation

$$
\begin{aligned}
\frac{\mathrm{d} E_{\text {kin }}}{\mathrm{d} t} & =\int \Gamma_{\text {turb }}^{E} \mathrm{~d}^{3} v r \mathrm{~d} r \mathrm{~d} \theta-e \int \partial_{t} \Phi F \mathrm{~d}^{3} v r \mathrm{~d} r \mathrm{~d} \theta \\
\frac{\mathrm{d} E_{\text {pol }}}{\mathrm{d} t} & =\int \frac{m \mathcal{N}}{B^{2}} \vec{\nabla}_{\perp} \Phi \cdot \partial_{t} \vec{\nabla}_{\perp} \Phi r \mathrm{~d} r \mathrm{~d} \theta+\int \frac{\mathcal{N} e^{2}}{T_{e}}\left(\Phi-\Phi_{\mathrm{FS}}\right) \partial_{t} \Phi r \mathrm{~d} r \mathrm{~d} \theta \\
\frac{\mathrm{d} E_{\text {turb }}}{\mathrm{d} t} & =\int q \frac{\partial_{r t}^{2} \Phi}{r B} \mathcal{I} \mathrm{d} \zeta r \mathrm{~d} r \mathrm{~d} \theta \\
\frac{\mathrm{d} E_{\text {tot }}}{\mathrm{d} t} & =\int \Gamma_{\text {turb }}^{E} \mathrm{~d}^{3} v r \mathrm{~d} r \mathrm{~d} \theta=0
\end{aligned}
$$

where we have used the Poisson equation 3.11 multiplied by $\partial_{t} \Phi$ to get the simplified last equation. The last equation corresponds to the energy exchange between the waves and the particles. For marginally stable modes, it is trivially zero. The conservation of the poloidal momentum can be computed directly from the Poisson equation 3.11

$$
\begin{aligned}
\frac{r^{2}}{q} m \mathcal{N} \partial_{t} u_{E} & =\frac{1}{r} \partial_{r} \int r v_{g}^{r} \mathcal{I} \mathrm{d} \zeta-e \frac{r}{q} \int \Gamma_{\mathrm{QL}}^{r} \mathrm{~d}^{3} v \\
& =-\frac{1}{r} \partial_{r} \int r \mathcal{W} \partial_{\zeta} \mathcal{D} \mathrm{d} \zeta
\end{aligned}
$$

where we have used the definition of the radial group velocity $v_{g}^{r}=-\partial_{\zeta} \mathcal{D} / \partial_{c} \mathcal{D}$. The first equality corresponds to the definition of the Reynolds stress as a flux of toroidal momentum, while the second equality allows to relate it to the potential spectrum.

\section{Drift wave model}

As a pedagogical example, let us first apply our approach to the wave-kinetic equation to the well-known Charney-Hasegawa-Mima model for slab drift waves (Charney \& Drazin 1961; Hasegawa \& Mima 1978). The advection equation for the vorticity $w(x, y)$ has a particularly simple form

$$
\partial_{t} w+\vec{v}_{E} \cdot \vec{\nabla} w+\beta \partial_{y} \phi=0
$$


We introduce the amplitude $\mathcal{A}$ and phase function as in equation 2.3

$$
\tilde{\phi}=\sqrt{2 \mathcal{A}} \exp \left(i \sigma(t, x)+i k_{y} y\right)
$$

Around a reference radial position $x$, with a background flow $u_{E}=\partial_{x} \Phi$ in the $y$ direction, the linearised response for 4.1 is easily computed as

$$
\tilde{w}=\frac{\beta-\partial_{x} W}{c+u_{E}} \tilde{\phi}
$$

with $W$ the equilibrium vorticity profile, and $c=-\partial_{t} \sigma / k_{y}$ the phase velocity in the $y$ direction. The action principle for Poisson equation becomes

$$
\begin{aligned}
\mathcal{S} & =\frac{1}{2} \int\left[\rho_{i}^{2}|\nabla \phi|^{2}+\tau|\phi|^{2}-\phi w[\phi]\right] \\
& =\int \mathcal{D}\left(-\frac{\partial_{t} \sigma}{k_{y}}+u_{E}, x, \frac{\partial_{x} \sigma}{k_{y}}, k_{y}\right) \mathcal{A} \\
\mathcal{D}(c, r, \zeta, n) & =k_{y}^{2} \rho_{i}^{2}\left(1+\zeta^{2}\right)+\tau-\frac{\beta-\partial_{x} W}{c}=0
\end{aligned}
$$

where $\zeta=\partial_{x} \sigma / k_{y}$. The $k_{y}^{2} \rho_{i}^{2}\left(1+\zeta^{2}\right)$ is the Laplacian operator in the Poisson equation. The dispersion relation for the drift-waves is $\mathcal{D}(c, r, \zeta, n)=0$. We introduce the Wigner function $\mathcal{W}$ and the wave action density $\mathcal{I}$

$$
\begin{aligned}
& \mathcal{W}=\int \phi(x+\delta x) \phi(x-\delta x) \mathrm{e}^{-i n \zeta x} n \mathrm{~d} x=2 \mathcal{A} \delta\left(\zeta-\frac{\partial_{x} \sigma}{n}\right) \\
& \mathcal{I}=\mathcal{W} \partial_{c} \mathcal{D}=\frac{\beta-\partial_{x} W}{c^{2}} \mathcal{W}=\frac{\left(\tau+k_{\perp}^{2} \rho_{i}^{2}\right)^{2}}{\beta-\partial_{x} W} \mathcal{W}
\end{aligned}
$$

By following the steps in paragraphs 2 and 3 , the wave-kinetic equation 2.13 and the Poisson equation 3.11 become

$$
\begin{aligned}
\partial_{t} \mathcal{I}-\partial_{x}\left[\frac{\partial_{\zeta} \mathcal{D}}{\partial_{c} \mathcal{D}} \mathcal{I}\right]+\partial_{\zeta}\left[\frac{\partial_{x} \mathcal{D}}{\partial_{c} \mathcal{D}} \mathcal{I}\right] & =0 \\
-\nabla^{2} \Phi & =\partial_{x} \int \mathcal{I} \mathrm{d} \zeta \mathrm{d} n
\end{aligned}
$$

with the group velocities given by

$$
\begin{aligned}
& v_{g}^{x}=-\frac{\partial_{\zeta} \mathcal{D}}{\partial_{c} \mathcal{D}}=-2 \zeta n^{2} \rho_{*}^{2} \frac{\beta-\partial_{x} W}{\left[n^{2} \rho_{*}^{2}\left(1+\zeta^{2}\right)+\tau\right]^{2}} \\
& v_{g}^{\zeta}=\frac{\partial_{x} \mathcal{D}}{\partial_{c} \mathcal{D}}=-\frac{\partial c}{\partial x}=-\partial_{x} u_{E}+\frac{\partial_{x}^{2} W}{n^{2} \rho_{*}^{2}\left(1+\zeta^{2}\right)+\tau}
\end{aligned}
$$

with $\rho_{*}=\rho_{i} / r$. The expression of the Reynolds stress is retrieved by considering the time evolution of $u_{E}$

$$
-\partial_{t} \partial_{x} \Phi=\partial_{x} \int \frac{\partial_{\zeta} \mathcal{D}}{\partial_{c} \mathcal{D}} \mathcal{I} \mathrm{d} \zeta \mathrm{d} n=\partial_{x} \int \rho_{*}^{2} n^{2} \zeta \mathcal{W} \mathrm{d} \zeta \mathrm{d} n
$$

We recover the conservation of the Wigner function of the vorticity, and not of the potential. This is consistent with the observations from the geometrical optics limit of the second cumulant expansion (Parker 2016, Gillot 2016). The formulation of the Reynolds stress exactly matches the expected $k_{x} k_{y}|\phi|^{2}$ from Euler equation, with less usual notations. Although derived from a different formalism, the obtained wave-kinetic system with velocity 4.6 features the saturation mechanism highlighted in Parker (2015); 
Ruiz et al. (2016). Its origin lies in the depletion of the free-energy source $\partial_{x} W=-\partial_{x}^{2} u_{E}$. The next section extends the physics application to the toroidal ITG mode.

\section{Generalised ITG model}

In order to avoid notation complexity, we consider a prototype generalised ITG model. The instability mechanism stems from the resonance between the toroidal phase velocity $c$ of the mode and the toroidal drift $\Omega_{d}$ of involved particles. In general, $\Omega_{d}$ is an even function of $\zeta$. For this reason, we shall take $\Omega_{d}=\Omega_{d 0}+\Omega_{d 1} \cos \zeta$, with $\Omega_{d 0}$ and $\Omega_{d 1}$ of the order of $u_{\mathrm{DT}}$. The dispersion relation writes

$$
\mathcal{D}(c, r, \zeta, n)=D\left(\frac{c+q u_{E}}{\Omega_{d}(\zeta)}, n\right)=0
$$

Without loss of generality, we suppose there is only one branch of solutions, $D\left(\delta_{n}+\right.$ $\left.i \varepsilon_{n}, n\right)=0$. In the converse case, the wave-kinetic system can be replaced by a sum over the branches. In this framework, one has

$$
c=\Omega_{d}(\zeta)\left(\delta_{n}+i \varepsilon_{n}\right)-q u_{E}
$$

Our derivation has been performed for ideal waves only, neglecting the wave-particle energy exchange. For consistency, we further assume no growth rate $\varepsilon_{n}=0$. The wavekinetic equation becomes

$$
\begin{aligned}
& \partial_{t} \mathcal{I}+\frac{1}{r} \partial_{r}\left(r v_{g}^{r} \mathcal{I}\right)+\partial_{\zeta}\left(\gamma_{E} \mathcal{I}\right)=0 \\
& v_{g}^{r}=\frac{\partial c}{q^{\prime} \partial \zeta}=-v_{g} \sin \zeta \\
& v_{g}^{\zeta}=-\frac{\partial c}{q^{\prime} \partial r}=\frac{\left(q u_{E}\right)^{\prime}}{q^{\prime}}=\gamma_{E}
\end{aligned}
$$

where the group velocity $v_{g}$ scales like the thermal magnetic drift. As expected, the zonal flow shear $\gamma_{E}$ acts on waves by an advection in $\zeta$ space.

\section{Effect of toroidicity on the zonostrophic instability}

Given these three equations 2.13, 3.1 and 3.11, we can discuss their behaviour around a plasma state $\left(\mathcal{F}_{\text {eq }}, \mathcal{I}_{\text {eq }}, \Phi_{\text {eq }}\right)$. For a small departure in the profiles and turbulence intensity, the coupled second-order system can be analysed linearly.

The wave-kinetic equation contains a radial advection 5.1 that scales like the magnetic drift. In certain conditions, this advection gives a travelling branch to the slab zonostrophic instability. Conversely to the slab model, the profiles in the toroidal case respond according to the GAM dynamics. Therefore, one can expect the GAM radial phase velocity and the wave-kinetic radial advection to resonate, destabilising the GAM mode.

Moreover, the radial velocity scales like equation 5.1. the inwards/outwards direction depends on the effective ballooning angle. With a background zonal flow shear, the turbulent spectrum is asymmetric in $\zeta$. This effect should allow one to explain the relation between the direction of avalanches and the sign of the zonal shear, as reported in numerical simulations (Idomura et al.|2009, McMillan et al. 2009). We take the coupled 
system as

$$
\begin{gathered}
\partial_{t} \mathcal{I}+\frac{1}{r} \partial_{r}\left(r v_{g}^{r} \mathcal{I}\right)+\partial_{\zeta}\left(\gamma_{E} \mathcal{I}\right)=0 \\
\partial_{t} \mathcal{F}(r, \theta, E, \mu)+\frac{v_{\|}}{q R} \partial_{\theta} \mathcal{F}-u_{D}\left(\cos \theta \partial_{\theta}+\sin \theta r \partial_{r}\right) \mathcal{F}+\frac{b}{B} \times \vec{\nabla} \Phi \cdot \vec{\nabla} \mathcal{F}=-\operatorname{div} \Gamma_{\mathrm{Q}}(6
\end{gathered}
$$

where we denote the magnetic drift angular velocity as

$$
u_{D}=\frac{m v_{\|}^{2}+\mu B}{e B R r}
$$

We chose to neglect the effect of the quasi-linear fluxes in 6.2 . These fluxes balance the excitation in the wave-kinetic equation. In order to keep the energetic consistency, we put this excitation to zero.

The coupled system 6.2 does not have any known self-consistent solution for non-zero background flow. Notably, numerical simulations feature strong radial corrugation of the zonal flow pattern. As a consequence, the application of the wave-kinetic method to those equilibria is not straightforward. More accurate descriptions of those equilibria are still under investigation (Kaw et al. 2001 ; Gürcan et al. 2009, Staebler et al. 2013 , Garbet et al. 2020), both on the general shape of the spectrum and on the zonal flow pattern. As a consequence, we avoid choosing such an equilibrium, and will only make use of selected components of the eventual equilibrium's turbulent spectrum.

We perturb the system with a fluctuation of the $n=0$ potential $\tilde{\Phi}$, with $p / r$ the radial mode number. Let $\omega$ be the mode frequency, and $\omega_{\|}=v_{||} / q R$. For simplicity, we neglect the back-action onto the density and temperature gradients used as free energy sources for the ITG turbulence. As a consequence, the growth of the mode in the thin corrugation limit — around the eddy size - may be overestimated (Parker 2016). In the Vlasov equation, we neglect the poloidal drifts (magnetic and $E \times B$ ) compared to the poloidal projection of the parallel velocity. We suppose the equilibrium electric field is purely radial $\Phi_{\text {eq }}(r)$. The Poisson equation is obtained from equation 3.11 .

$$
\begin{aligned}
-i \omega \tilde{\mathcal{I}}-i p \frac{v_{g}}{r} \sin \zeta \tilde{\mathcal{I}}+\gamma_{E} \partial_{\zeta} \tilde{\mathcal{I}} & =\frac{p^{2}}{r^{2}} \frac{\tilde{\Phi}_{0}}{B} \partial_{\zeta} \mathcal{I}_{\mathrm{eq}} \\
-i \omega \tilde{F}+\omega_{\|} \partial_{\theta} \tilde{F}-u_{D} \sin \theta r \partial_{r} \tilde{F} & =-\left[\omega_{\|} \partial_{\theta}-u_{D} \sin \theta r \partial_{r}\right] \tilde{\Phi} \frac{\mathcal{F}_{\mathrm{eq}} e}{T_{\mathrm{eq}}} \\
\left(-p^{2} \rho_{*}^{2}+\rho_{*}^{2} \partial_{\theta}^{2}+\tau\right) \frac{\mathcal{N}_{\mathrm{eq}} e^{2}}{T_{\mathrm{eq}}} \tilde{\Phi} & =e \int \tilde{F}+i p \int \frac{q \tilde{\mathcal{I}}}{r^{2} B} \mathrm{~d} \zeta
\end{aligned}
$$

where we use the local normalised Larmor radius $\rho_{*}=\rho_{i} / r$. We denote as $\Phi_{0, c, s}$ the symmetric, cosine and sine components of $\tilde{\Phi}$, likewise for $\tilde{F}$. We perform a similar decomposition for $\tilde{\mathcal{I}}$ with the ballooning angle $\zeta$.

$$
\begin{aligned}
& \left(\begin{array}{ccc}
-2 i \omega & -i p \frac{v_{g}}{r} & 0 \\
-i p \frac{v_{g}}{r} & -i \omega & -\gamma_{E} \\
0 & +\gamma_{E} & -i \omega
\end{array}\right)\left(\begin{array}{l}
\tilde{\mathcal{I}}_{0} \\
\tilde{\mathcal{I}}_{s} \\
\tilde{\mathcal{I}}_{c}
\end{array}\right)=\frac{p^{2}}{r^{2}} \frac{\tilde{\Phi}_{0}}{B}\left(\begin{array}{c}
0 \\
-\mathcal{I}_{\mathrm{eq}, c} \\
\mathcal{I}_{\mathrm{eq}, s}
\end{array}\right) \\
& \left(\begin{array}{ccc}
-2 i \omega & -i p u_{D} & 0 \\
-i p u_{D} & -i \omega & -\omega_{\|} \\
0 & +\omega_{\|} & -i \omega
\end{array}\right)\left(\begin{array}{c}
\tilde{F}_{0} \\
\tilde{F}_{s} \\
\tilde{F}_{c}
\end{array}\right)=\frac{\mathcal{F}_{\mathrm{eq}} e}{T_{\mathrm{eq}}}\left(\begin{array}{ccc}
0 & i p u_{D} & 0 \\
i p u_{D} & 0 & -\omega_{\|} \\
0 & +\omega_{\|} & 0
\end{array}\right)\left(\begin{array}{c}
\tilde{\Phi}_{0} \\
\tilde{\Phi}_{s} \\
\tilde{\Phi}_{c}
\end{array}\right)(6.7)
\end{aligned}
$$

We can verify that the matrices on the left-hand side are skew-symmetric. This is consistent with the advection form of the Vlasov and wave-kinetic equations. For simplicity, we set the wave group velocity as $v_{g}=u_{\mathrm{DT}} / q^{\prime}$, with the thermal toroidal 
magnetic drift frequency $u_{\mathrm{DT}}=q T / e B R r$. Let $u_{\mathrm{TR}}$ be the poloidal transit frequency, $u_{\mathrm{TR}}=v_{\mathrm{th}} / q R$. We introduce the normalised mode frequency $\Omega=\frac{\omega}{u_{\mathrm{TR}} \sqrt{2}}$, mode number $P=\frac{u_{\mathrm{DT}}}{2 u_{\mathrm{TR}} q} p=q \rho_{*} p / 2$, and background flow shear $S=\frac{\gamma_{E}}{u_{\mathrm{TR}} \sqrt{2}}$. Inverting the two matrices gives the resolved form

$$
\begin{aligned}
& \tilde{\mathcal{I}}_{0}=\frac{i P^{3} / s}{\Omega^{2}-S^{2}-P^{2} / s^{2}}\left[\mathcal{I}_{\mathrm{eq}, c}-i \frac{S}{\Omega} \mathcal{I}_{\mathrm{eq}, s}\right] \frac{2 \tilde{\Phi}_{0}}{q^{2} \rho_{*}^{2} r^{2} u_{\mathrm{TR}} B} \\
& \tilde{F}_{0}=\frac{e \mathcal{F}_{\mathrm{eq}}}{2 T_{\mathrm{eq}}} \frac{-\Omega P \sqrt{2} \frac{q u_{D}}{u_{\mathrm{DT}}} \tilde{\Phi}_{s}+2 P^{2}\left(\frac{q u_{D}}{u_{\mathrm{DT}}}\right)^{2} \tilde{\Phi}_{0}}{\Omega^{2}-\Omega_{\|}^{2}} \\
& \tilde{F}_{s}=\frac{e \mathcal{F}_{\mathrm{eq}}}{T_{\mathrm{eq}}} \frac{-\sqrt{2} \Omega P \frac{q u_{D}}{u_{\mathrm{DT}}} \tilde{\Phi}_{0}+\Omega_{\|}^{2} \tilde{\Phi}_{s}+i \Omega \Omega_{\|} \tilde{\Phi}_{c}}{\Omega^{2}-\Omega_{\|}^{2}}
\end{aligned}
$$

with $s=r q^{\prime} / q$ the magnetic shear. For a symmetric distribution function, the only the even terms in $\Omega_{\|}$contribute, so $\tilde{\Phi}_{c}$ disappears from the description. Integrating in velocity, we obtain

$$
\begin{aligned}
& \tilde{N}_{0}=\frac{e \mathcal{N}_{\mathrm{eq}}}{2 T_{\mathrm{eq}}}\left[P I_{1} \tilde{\Phi}_{s}+P^{2} I_{2} \tilde{\Phi}_{0}\right] \\
& \tilde{N}_{s}=\frac{e \mathcal{N}_{\mathrm{eq}}}{T_{\mathrm{eq}}}\left[P I_{1} \tilde{\Phi}_{0}+I_{3} \tilde{\Phi}_{s}\right]
\end{aligned}
$$

where we used the resonant integrals from Girardo (2015):

$$
\begin{aligned}
& I_{1}=\sqrt{2} \int \frac{\Omega \frac{q u_{D}}{u_{\mathrm{DT}}}}{\Omega^{2}-\Omega_{\|}^{2}} \frac{\mathcal{F}_{\mathrm{eq}}}{\mathcal{N}_{\mathrm{eq}}}=-\sqrt{2}\left[Z[\Omega]\left(\frac{1}{2}+\Omega^{2}\right)+\Omega\right] \\
& I_{2}=2 \int \frac{\left(\frac{q u_{D}}{u_{\mathrm{DT}}}\right)^{2}}{\Omega^{2}-\Omega_{\|}^{2}} \frac{\mathcal{F}_{\mathrm{eq}}}{\mathcal{N}_{\mathrm{eq}}}=-2\left[Z[\Omega]\left(\frac{1}{2 \Omega}+\Omega+\Omega^{3}\right)+\frac{3}{2}+\Omega^{2}\right] \\
& I_{3}=\int \frac{\Omega_{\|}^{2}}{\Omega^{2}-\Omega_{\|}^{2}} \frac{\mathcal{F}_{\mathrm{eq}}}{\mathcal{N}_{\mathrm{eq}}}=-1-\Omega Z[\Omega]
\end{aligned}
$$

Finally, Poisson equation gives

$$
\begin{aligned}
\frac{P^{2}}{q^{2}} \tilde{\Phi}_{0} & =\frac{1}{2}\left[P I_{1} \tilde{\Phi}_{s}+P^{2} I_{2} \tilde{\Phi}_{0}\right]+i \frac{2 P}{r^{2} \rho_{*} B} \frac{T_{\mathrm{eq}}}{\mathcal{N}_{\mathrm{eq}} e^{2}} \tilde{\mathcal{I}}_{0} \\
& =\frac{1}{2}\left[P I_{1} \tilde{\Phi}_{s}+P^{2} I_{2} \tilde{\Phi}_{0}\right] \\
& -4 \tilde{\Phi}_{0} \frac{P^{4} / s}{\Omega^{2}-S^{2}-P^{2} / s^{2}} \int \frac{\mathcal{I}_{\mathrm{eq}, c}-i \frac{S}{\Omega} \mathcal{I}_{\mathrm{eq}, s}}{\mathcal{N}_{\mathrm{eq}} m r^{2} u_{\mathrm{DT}}} \mathrm{d} \zeta \\
\left(\tau+\rho_{*}^{2}+\frac{P^{2}}{q^{2}}\right) \tilde{\Phi}_{s} & =P I_{1} \tilde{\Phi}_{0}+I_{3} \tilde{\Phi}_{s}
\end{aligned}
$$

We introduce the turbulent intensity as $\mathcal{T}_{c, s}=\frac{\int \mathcal{I}_{\mathrm{eq}, c, s} \mathrm{~d} \zeta}{\mathcal{N}_{\mathrm{eq}} m r^{2} u_{\mathrm{DT}}}$. The dispersion relation is 
given by $D_{G}(\Omega)=0$ with

$$
\begin{aligned}
D_{G}(\Omega) & =\frac{1}{q^{2}}-\frac{J_{2}(\Omega)}{2}-\frac{I_{1}^{2}(\Omega)}{2\left(\tau+\frac{P^{2}}{q^{2}}-I_{3}(\Omega)\right)} \\
J_{2} & =I_{2}-\frac{8 P^{2} / s}{\Omega^{2}-S^{2}-P^{2} / s^{2}}\left[\mathcal{T}_{c}-i \frac{S}{\Omega} \mathcal{T}_{s}\right]
\end{aligned}
$$

The dispersion relation 6.18 is plotted figure 1 . This plot is done with $q=1.6, s=1$ without any sheared flow $S=0$. The turbulent intensity is chosen as $\mathcal{T}_{c}=10^{-2}$ and $\mathcal{T}_{s}=0$. This corresponds to an in-out asymmetry of turbulent energy of the order of $\mathcal{T}_{c} q^{2} \rho_{*}^{2} \varepsilon^{2}$ times the plasma pressure. For $P=0$, we recover the usual GAM dispersion relation, with $J_{2}$ becoming $I_{2}$ :

$$
D_{\mathrm{GAM}}(\Omega, P, \mathcal{T})=\frac{1}{q^{2}}-\frac{I_{2}}{2}-\frac{I_{1}^{2}}{2\left(\tau+\frac{P^{2}}{q^{2}}-I_{3}\right)}
$$

The GAM is located at $\Omega_{\mathrm{GAM}}=3.1-0.02 i$. For $P / s$ far from $\Omega_{\mathrm{GAM}}$, the zero due to the zonostrophic instability provides an unstable mode with growth rate $\Gamma=0.01$, and is located near the resonance position $\Omega=P / s$. For $P=3$, the two zeros interact. The zonostrophic instability is further destabilised at $\Omega=2.8+0.35 i$, while the GAM is strongly damped at $\Omega=2.8-0.38 i$. This example does not contain a linear growth rate for the turbulent structures, so the growth of the zonal flow comes from pumping energy from turbulence.

It is straightforward to extend the relation dispersion 6.18 to handle EGAMs (Girardo 2015). The same plot can be done with a population of $7 \%$ of energetic particles going at 2.8 times the thermal velocity, figure 2. The EGAM lies at $\Omega=2.5+0.07 i$. For $P=2.5$, the zonostrophic instability interacts with it. The EGAM is stabilised at $\Omega=$ $2.4-0.17 i$, while the zonostrophic mode is destabilised to $\Omega=2.3+0.24 i$. This behaviour is consistent with the observations from Zarzoso et al. $(2013)$ : the avalanche synchronises at the EGAM frequency when energetic particles are present.

The coupled instability develops at a resonance between the GAM frequency and the turbulent radial group velocity. The resonant radial wavenumber $k_{r}$ is a few times $s / q^{2} \rho_{i}$. For weak magnetic shear, $k_{r} \rho_{i}$ is a fraction of a unit. The ordering assumption on the radial wavenumber is not ensured. Such inconsistency is typical of the wave-kinetic model (Parker 2016). In addition, the quasi-linear framework on which wave-kinetic theory is based is known for its relevance well beyond its validity limits (G. Laval \& Adam 2018).

We emphasize the unstable mode in figures 1 and 2 is not the GAM, but rather the zonostrophic mode which has been pushed-up by the GAM (although the converse can actually happen for EGAMs depending on the plasma parameters). Furthermore, being a resonance process, the destabilisation by the GAM weakens when the GAM damping increases. For GAMs with a high enough damping rate this mechanism may not operate at all. Similarly, when the GAM's damping rate depends on the radial position, the dynamics could select the most unstable growth rate for an enlarged radial span, and ensure an undamaged propagation through non-linear effects. In this light, we interpret the results from Villard et al. (2019) as a destabilisation of our unstable mode close to the edge, at the edge GAM frequency. This mode would propagate inwards from the edge, keeping this edge GAM frequency.

In contrast to the slab drift-wave problem, the unstable modes featured in the dispersion relation 6.18 have bounded growth rate, even for high wavenumbers. This can be 

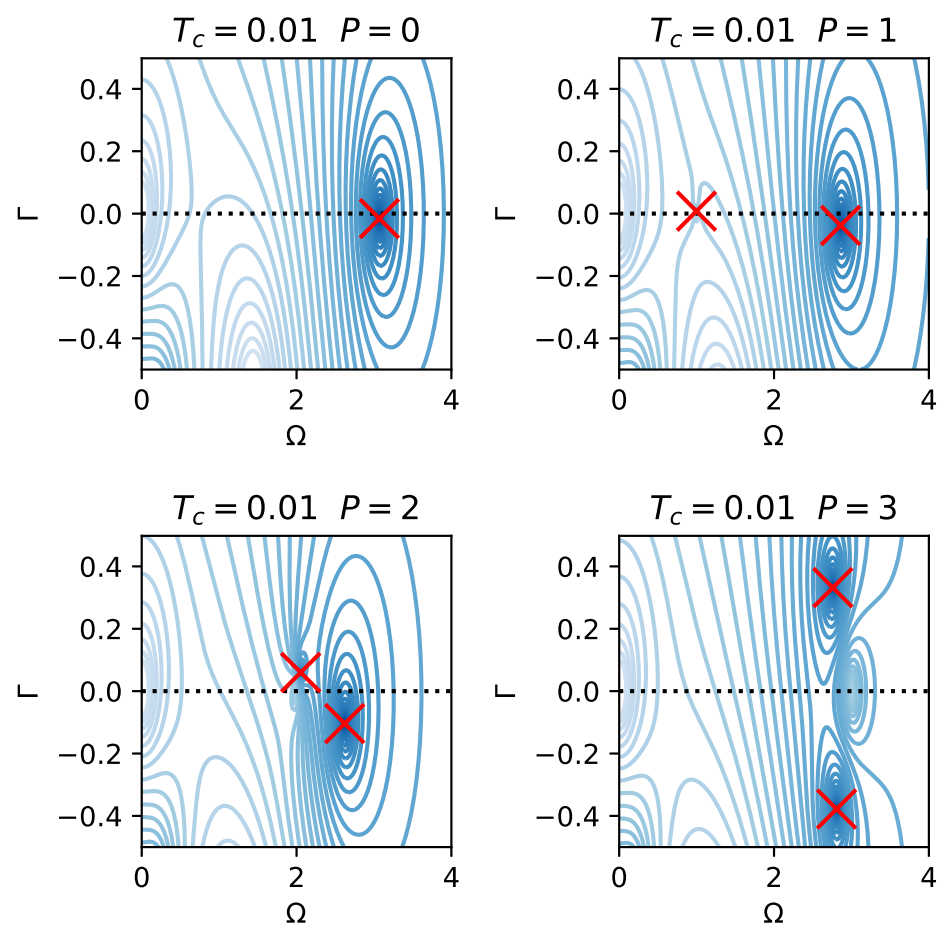

Figure 1. Contour lines of $\left|D_{G}\right|$ for $q=1.6, \tau=1$ and $s=1 . S=0$. The GAM frequency is $\Omega=3.07-0.02 i$. The zeros are in dark blue. The zeros due to the zonostrophic instability is highlighted by a red cross.

shown easily by considering the limit of large growth rate as

$$
\begin{aligned}
D_{G}(\Omega \rightarrow \infty) & \approx \frac{1}{q^{2}}-\frac{J_{2}(\Omega)}{2}-\frac{1}{\Omega^{2} \tau+\Omega^{2} \frac{P^{2}}{q^{2}}-1 / 2} \\
J_{2}(\Omega \rightarrow \infty) & \approx \frac{2}{\Omega^{2}}-\frac{8 P^{2} / s}{\Omega^{2}-P^{2} / s^{2}}\left[\mathcal{T}_{c}-i \frac{S}{\Omega} \mathcal{T}_{s}\right]
\end{aligned}
$$

Solving this dispersion relation reduces to the roots of the following quartic equation

$$
\Omega^{4} P^{2} s^{2}-\Omega^{2}\left(P^{4}+P^{2} q^{2} s^{2}+4 P^{4} q^{2} \mathcal{T}_{c}+q^{4} s^{2}\right)+P^{4} q^{2}+P^{2} q^{4}=0
$$

which has four real roots for $P$ large enough. As a consequence, the approximation of static free-energy sources is acceptable, even in the thin-corrugation limit. This contrasts with the situtation of slab drift-waves where Parker (2016) found this approximation to lead to unphysical results.

If we add a non-zero background zonal shear, we expect the system to develop an asymmetry depending on the sign of $\Omega$. This is not the case without a turbulent growth rate. Turbulent structures are allowed to travel the full poloidal plane. The system is symmetric in phase velocity, and does not prefer a direction over the other. This is a consequence of the joint symmetry principle (Hwa \& Kardar 1992, Diamond \& Hahm 1995). In order to regain the asymmetry, we need to take into account the differential growth between the two sides. The damping on the high-field side cuts the poloidal travel of the turbulent structures. We add a growth rate $\gamma=\alpha \cos \zeta$ to equation 6.1 . The 

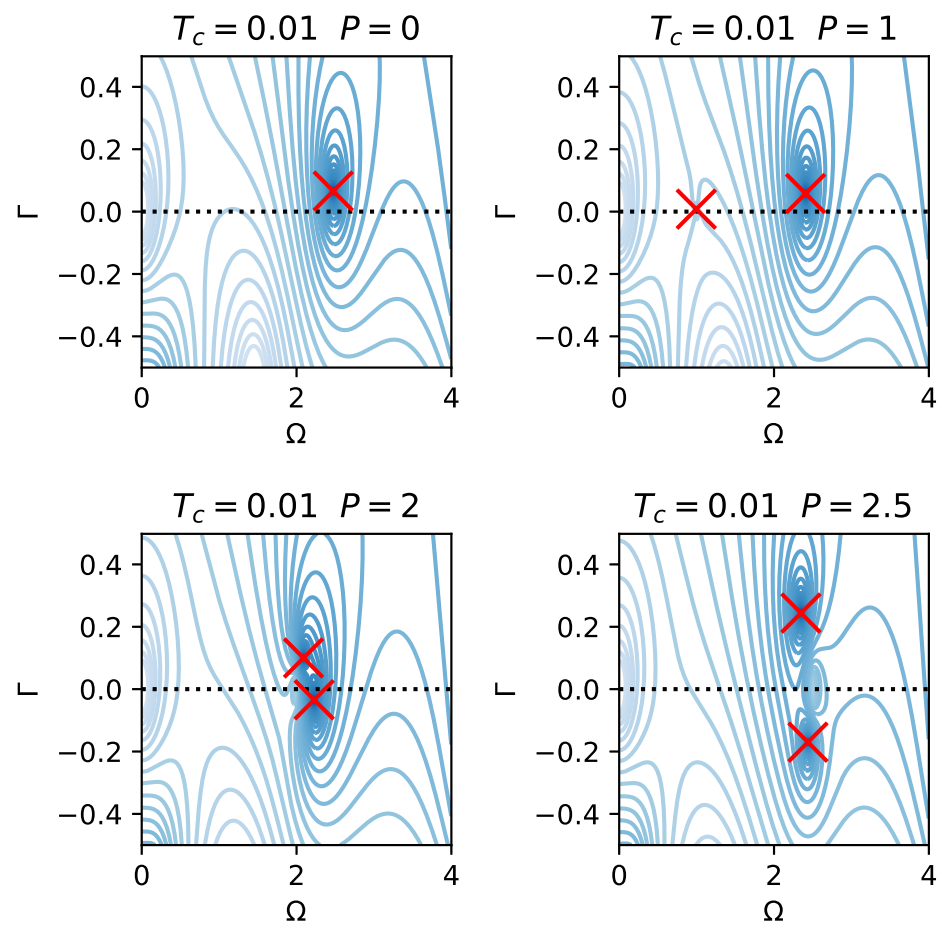

Figure 2. Contour lines of $\left|D_{G}\right|$ for $q=1.6, \tau=1$ and $s=1$ with $7 \%$ energetic particles at 2.8 times the thermal velocity. $S=0$. The EGAM frequency is $\Omega=2.47+0.07 i$. The zeros are in dark blue. The zeros due to the zonostrophic instability is highlighted by a red cross.

$\alpha$ coefficient constrains a localised growth of the turbulence on the low-field-side. This effectively expresses the intensity growth where the instability growth is maximum. The $J_{2}$ function becomes

$$
J_{2}=I_{2}-\frac{P^{2} / s}{\Omega^{2}+2 A^{2}-S^{2}-P^{2} / s^{2}}\left[\left(1-\frac{A S}{\Omega P}\right) \mathcal{T}_{c}-i\left(\frac{S}{\Omega}-\frac{A}{P}\right) \mathcal{T}_{s}\right]
$$

where $A=\alpha / u_{\mathrm{TR}}$. By introducing $A \neq 0$, the structures are damped when arriving at the high-field side. The symmetry between $\zeta>0$ and $\zeta<0$ is broken, and the instability can develop with a preferred direction. We consider this modified system with $A=S=2$. The dispersion relation for $P=3$ is shown figure 3 . The two instabilities are located at $\Omega=-2.3+0.41 i$ and $\Omega=2.3+0.11 i$. The direction is consistent with the observed inwards avalanches for positive zonal flow shear. Contrary to Sasaki et al. $(2018 a)$, we do not need to introduce an ad-hoc up-down asymmetry of the turbulent spectrum, it is generated self-consistently by the ballooned growth rate and the background flow shear. Nevertheless, such an asymmetry should be expected for tokamaks with realistic magnetic geometry, and would modify the directionality of avalanches as emphasised in Hager \& Hallatschek (2012). Although the wave-particle energy exchange is not self-consistent for $\alpha \neq 0$, the instability mechanism already exists in the self-consistent $\alpha=0$ case. As a result, the computed instability is not spurious, but a modification of the former case. 


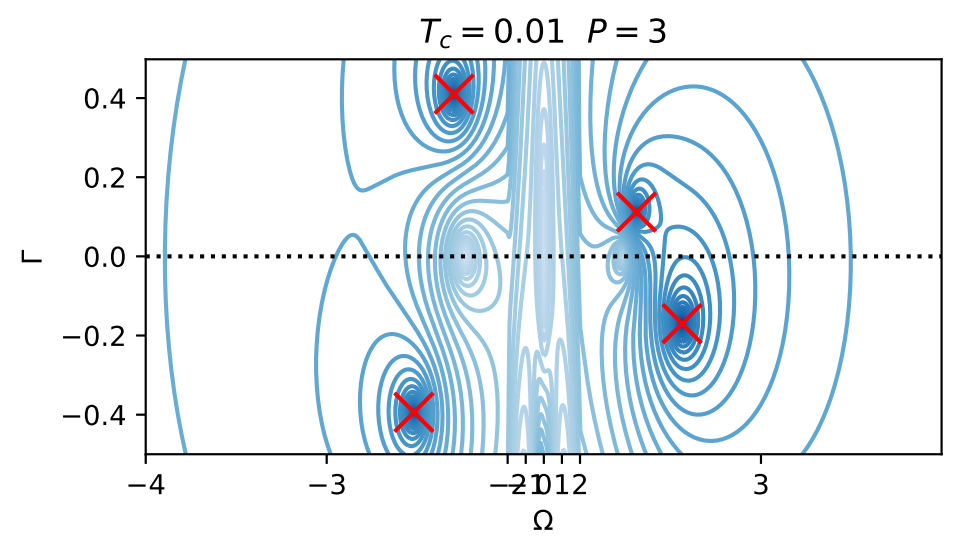

Figure 3. Contour lines of $\left|D_{G}\right|$ for $q=1.6, \tau=1$ and $s=1 . A=S=2$. The unstable inwards mode $\Omega<0$ is more unstable than the outwards one. The $-2<\Omega<2$ region has been compressed to help visualisation in the $2<|\Omega|<4$ regions.

\section{Conclusion}

In order to study the zonostrophic instability in toroidal plasmas, we developed a self-consistent and conservative formulation of the wave-kinetic equation coupled to a background plasma. This formulation is parameterised by the dispersion relation for the underlying turbulent linear wave, but is restricted to marginally stable modes. It is usable both in slab and toroidal geometry. This conservative formulation has been used to investigate the effect of toroidal geometry on the generation of zonal flows.

In toroidal geometry, zonal flows affect turbulent cells by moving them toroidally, and by shearing them. This shearing acts by moving the turbulent cells in the poloidal direction, making them ballooned above or below the mid-plane. As the toroidal drift velocity depends on the ballooning angle of turbulence, the ITG mode frequency follows the same dependency. Since the ballooning angle is related to the radial mode number of the turbulent cell, this induces a radial group velocity of the turbulent cells, mostly following the ion direction.

The generic zonostrophic instability carries over from slab to toroidal geometry. It is driven by the modulation of the drift-wave turbulence by a sheared zonal flow. This generic instability has its phase velocity close to the radial group velocity of the underlying turbulence. In toroidal geometry, the zonal flow responds according to the GAM dynamics, with a specific radial phase velocity. When the radial motion of turbulent cells resonates with the GAM radial phase velocity, the zonostrophic instability and the GAM interact. The zonostrophic instability is further destabilised, and the GAM has stronger damping. This mechanism could be responsible for the avalanche behaviour. It is able to explain the typical frequency of avalanches, close to the GAM frequency. Furthermore, it is able to reproduce the synchronisation to EGAM frequency (Zarzoso et al. 2013). When a background zonal flow shear is present, a ballooned turbulence has an up-down asymmetry. The footprint of this asymmetry gets carried over to the avalanches by preferring the same propagation direction. Additional sources of asymmetry are to be expected in more realistic magnetic configurations (Hager \& Hallatschek 2012).

The resulting coupled instability develops at a resonance between the GAM frequency and the radial magnetic drift. As a consequence, the resonant radial wavenumber $k_{r}$ is a few times $s / q^{2} \rho_{i}$. In the weak magnetic shear regime, $k_{r} \rho_{i}$ is a fraction of a unit. 
As a consequence, the radial scale separation between turbulence and GAMs is only marginally verified. Such inconsistency between the derivation and the application is typical of the wave-kinetic model (Parker 2016). Slower branches of GAMs, like trapped particles driven and precession driven (Sasaki et al.|2016), may provide a more reasonable radial scale.

The non-linear regime with an established GAM has been described by Sasaki (2018); Sasaki et al. $(2018 b a)$. An extreme in the flow can act as a trap in phase space for turbulent cells. The toroidal drift wave phase velocity can be approximated as

$$
c \approx-q u_{E}+\delta_{n} \Omega_{d}(\zeta) \approx c_{0}-\frac{1}{2}\left(q u_{E}\right)^{\prime \prime}\left(r-r_{0}\right)^{2}-\delta_{n} \Omega_{d}(\zeta) \frac{\zeta^{2}}{2}
$$

For maxima of the flow, $\left(q u_{E}\right)^{\prime \prime}>0$, the phase velocity is a potential well, turbulent structures may become trapped. On the contrary, for minima of the flow, $\left(q u_{E}\right)^{\prime \prime}<0$, the phase velocity has a saddle point, turbulent structures are expelled. This asymmetry has been observed in numerical simulations for both gyro-kinetic toroidal systems (DifPradalier et al. |2015, McMillan et al. 2011; Zhu et al. 2018b; Ivanov et al. 2020, Gillot 2020). The same generic mechanism exists for stationary zonal flow (Zhu et al. 2020): the stability of the zonal flow pattern depends on the sign of the curvature. The formalism developed in this article could be extended to account for these additional phenomena. These trapped eikonal waves actually correspond to stationary radiallycoherent waves inside the trap (McDonald 1988). Lifting the eikonal hypothesis may provide useful insight into the nature of non-linear cooperative structures between GAMs and turbulence.

\section{Acknowledgements}

The authors want to thank Ph. Ghendrih, V. Grandgirard, Ch. Passeron, and acknowledge stimulating discussions with the participants at the 2019 Festival de Théorie in Aixen-Provence. This work has been carried out within the framework of the EUROfusion Consortium and was supported by the EUROfusion - Theory and Advanced Simulation Coordination (E-TASC) initiative under the TSVV (Theory, Simulation, Verification and Validation) "L-H transition and pedestal physics" project (2019-2020). It has also received funding from the Euratom research and training program. The authors gratefully acknowledge funding from the European Commission Horizon 2020 research and innovation program under Grant Agreement No. 824158 (EoCoE-II). The views and opinions expressed herein do not necessarily reflect those of the European Commission. This work was also granted access to the HPC resources of TGCC and CINES made by GENCI, and to the EUROfusion High Performance Computer Marconi-Fusion. We acknowledge PRACE for awarding us access to Joliot-Curie at GENCI@CEA, France and MareNostrum at Barcelona Supercomputing Center (BSC), Spain.

\section{REFERENCES}

Adam, J. C., Laval, G. \& Pesme, D. 1979 Reconsideration of quasilinear theory. Physical Review Letters 43 (22), 1671-1675.

Anderson, J., Nordman, H., Singh, R. \& Weiland, J. 2002 Zonal flow generation in ion temperature gradient mode turbulence. Physics of Plasmas 9 (11), 4500-4506.

Anderson, J., Nordman, H., Singh, R. \& Weiland, J. 2006 Zonal flow generation in collisionless trapped electron mode turbulence. Plasma Physics and Controlled Fusion 48 (5), 651-661. 
Bak, P., TAng, C. \& Wiesenfeld, K. 1987 Self-organized criticality: An explanation of the 1/f noise. Physical Review Letters 59 (4), 381-384.

Beyer, P., Benkadda, S., Garbet, X. \& Diamond, P. H. 2000 Nondiffusive transport in tokamaks: Three-dimensional structure of bursts and the role of zonal flows. Physical Review Letters 85 (23), 4892-4895.

Brillouin, L. 1960 Wave propagation and group velocity. Academic Press, New York.

Brizard, A. J. \& Tronko, N. 2011 Exact momentum conservation laws for the gyrokinetic vlasov-poisson equations. Physics of Plasmas 18 (8), 082307, arXiv: 1105.1145v2.

CAndy, J. \& WAltz, R. E. 2003 Anomalous transport scaling in the DIII-D tokamak matched by supercomputer simulation. Physical Review Letters 91 (4).

Carreras, B. A., Hidalgo, C., Sánchez, E., Pedrosa, M. A., Balbín, R., GarcíaCortés, I., van Milligen, B., Newman, D. E. \& Lynch, V. E. 1996 Fluctuationinduced flux at the plasma edge in toroidal devices. Physics of Plasmas 3 (7), 2664-2672.

Champeaux, S. \& Diamond, P. H. 2001 Streamer and zonal flow generation from envelope modulations in drift wave turbulence. Physics Letters A 288 (3-4), 214-219.

Charney, J. G. \& Drazin, P. G. 1961 Propagation of planetary-scale disturbances from the lower into the upper atmosphere. Journal of Geophysical Research 66 (1), 83-109.

Citrin, J., Bourdelle, C., Casson, F. J., Angioni, C., Bonanomi, N., Camenen, Y., Garbet, X., Garzotti, L., Görler, T., Gürcan, O., Koechl, F., Imbeaux, F., Linder, O., van de Plassche, K., Strand, P. \& And, G. S. 2017 Tractable fluxdriven temperature, density, and rotation profile evolution with the quasilinear gyrokinetic transport model QuaLiKiz. Plasma Physics and Controlled Fusion 59 (12), 124005.

Diamond, P. H. \& HAнM, T. S. 1995 On the dynamics of turbulent transport near marginal stability. Physics of Plasmas 2 (10), 3640-3649.

Diamond, P. H., Iтон, S.-I., Iтон, K. \& Haнm, T. S. 2005 Zonal flows in plasma-a review. Plasma Physics and Controlled Fusion 47 (5), 35-161.

Dif-Pradalier, G., Hornung, G., Garbet, X., Ghendrih, P., Grandgirard, V., Latu, G. \& Sarazin, Y. 2017 The $\mathbf{E} \times \mathbf{B}$ staircase of magnetised plasmas. Nuclear Fusion 57 (6), 066026.

Dif-Pradalier, G., Hornung, G., Ghendrih, P., Sarazin, Y., Clairet, F., Vermare, L., Diamond, P. H., Abiteboul, J., Cartier-Michaud, T., Ehrlacher, C., Estève, D., Garbet, X., Grandgirard, V., Gürcan, Ö. D., Hennequin, P., Kosuga, Y., Latu, G., Maget, P., Morel, P., Norscini, C., Sabot, R. \& Storelli, A. 2015 Finding the elusive $\mathbf{E} \times \mathbf{B}$ staircase in magnetized plasmas. Physical Review Letters 114 (8).

Dodin, I. Y. \& Fisch, N. J. 2012 Adiabatic nonlinear waves with trapped particles. I. general formalism. Physics of Plasmas 19 (1), 012102.

FARrell, B. F. \& IOANnOU, P. J. 2007 Structure and spacing of jets in barotropic turbulence. Journal of the Atmospheric Sciences 64 (10), 3652-3665.

G. Laval, D. P. \& AdAm, J.-C. 2018 Wave-particle and wave-wave interactions in hot plasmas: a french historical point of view. The European Physical Journal H 43 (4-5), 421-458.

Garbet, X., Sarazin, Y., Beyer, P., Ghendrih, P., Waltz, R., Ottaviani, M. \& Benkadda, S. 1999 Flux driven turbulence in tokamaks. Nuclear Fusion 39, 2063-2068.

Garbet, X., Sarazin, Y., Ghendrih, P., Benkadda, S., Beyer, P., Figarella, C. \& Voitsekhovitch, I. 2002 Turbulence simulations of transport barriers with toroidal velocity. Physics of Plasmas 9 (9), 3893-3905.

Garbet, X., Varennes, R., Gillot, C., Dif-Pradalier, G., Sarazin, Y., Bourne, E., Grandgirard, V., Ghendrih, P., Zarzoso, D. \& L.Vermare 2020 Zonal instability and wave trapping .

Gillot, C. 2016 Study of the interaction between drift wave turbulence and zonal flows in a magnetic confinement device. Master's thesis, Université Paris-Saclay.

Gillot, C. 2020 Model reduction for tokamak plasma turbulence : beyond fluid and quasi-linear descriptions. $\mathrm{PhD}$ thesis.

Girardo, J.-B. 2015 Control of instabilities and turbulence by fast particles in fusion plasmas. Theses, Ecode doctorale de l'Ecole Polytechnique (EDX).

Gürcan, O. D., Garbet, X., Hennequin, P., Diamond, P. H., Casati, A. \& Falchetto, G. 2009 Wave-number spectrum of drift-wave turbulence 102 (25).

Görler, T., Lapillonne, X., Brunner, S., Dannert, T., Jenko, F., Aghdam, S. K., 
Marcus, P., McMillan, B. F., Merz, F., Sauter, O., Told, D. \& Villard, L. 2011 Flux- and gradient-driven global gyrokinetic simulation of tokamak turbulence. Physics of Plasmas 18 (5), 056103.

Hager, R. \& HallatscheK, K. 2012 The nonlinear dispersion relation of geodesic acoustic modes. Physics of Plasmas 19 (8), 082315.

HallatscheK, K. \& Biskamp, D. 2001 Transport control by coherent zonal flows in the core/edge transitional regime. Physical Review Letters 86 (7), 1223-1226.

Hasegawa, A. \& Mima, K. 1978 Pseudo-three-dimensional turbulence in magnetized nonuniform plasma. Physics of Fluids 21 (1), 87.

Hwa, T. \& KARDAR, M. 1992 Avalanches, hydrodynamics, and discharge events in models of sandpiles. Physical Review A $4 \mathbf{5}$ (10), 7002-7023.

Idomura, Y., Urano, H., Aiba, N. \& Tokuda, S. 2009 Study of ion turbulent transport and profile formations using global gyrokinetic full- $f$ Vlasov simulation. Nuclear Fusion 49 (6), 065029.

Ivanov, P. G., Schekochinin, A. A., Dorland, W., Field, A. R. \& Parra, F. I. 2020 Zonally dominated dynamics and dimits threshold in curvature-driven ITG turbulence. Journal of Plasma Physics 86 (5), arXiv: 2004.04047.

Jimenez, J. \& Whitham, G. B. 1976 An averaged Lagrangian method for dissipative wavetrains. Proceedings of the Royal Society of London. A. Mathematical and Physical Sciences 349 (1658), 277-287.

Kaufman, A. N., Ye, H. \& Hui, Y. 1987 Variational formulation of covariant eikonal theory for vector waves. Physics Letters A 120 (7), 327-330.

KaW, P., Singh, R. \& Diamond, P. H. 2001 Coherent nonlinear structures of drift wave turbulence modulated by zonal flows. Plasma Physics and Controlled Fusion 44 (1), 5159.

Landau, L. D. \& Lifschitz, E. M. 1984 Electrodynamics of continuous media. Oxford Oxfordshire New York: Pergamon.

Lin, Z., Ethier, S., Hahm, T. S. \& TAng, W. M. 2002 Size scaling of turbulent transport in magnetically confined plasmas. Physical Review Letters 88 (19).

MCDonald, S. W. 1988 Phase-space representations of wave equations with applications to the eikonal approximation for short-wavelength waves. Physics Reports 158 (6), 337-416.

McDonald, S. W. 1991 Wave kinetic equation in a fluctuating medium. Physical Review A 43 (8), 4484-4499.

McDonald, S. W., Grebogi, C. \& Kaufman, A. N. 1985 Locally coupled evolution of wave and particle distribution in general magnetoplasma geometry. Physics Letters A 111 (1-2), $19-21$.

McDonald, S. W. \& Kaufman, A. N. 1985 Weyl representation for electromagnetic waves: The wave kinetic equation. Physical Review A 32 (3), 1708-1713.

McMillan, B. F., Hill, P., Bottino, A., Jolliet, S., Vernay, T. \& Villard, L. 2011 Interaction of large scale flow structures with gyrokinetic turbulence. Physics of Plasmas 18 (11), 112503.

McMillan, B. F., Jolliet, S., Tran, T. M., Villard, L., Bottino, A. \& Angelino, P. 2009 Avalanchelike bursts in global gyrokinetic simulations. Physics of Plasmas 16 (2), 022310 .

McMillan, B. F., Pringle, C. C. T. \& Teaca, B. 2018 Simple advecting structures and the edge of chaos in subcritical tokamak plasmas. Journal of Plasma Physics 84 (6).

Moyal, J. E. \& Bartlett, M. S. 1949 Quantum mechanics as a statistical theory. Mathematical Proceedings of the Cambridge Philosophical Society 45 (01), 99.

Newman, D. E., Carreras, B. A., Diamond, P. H. \& Hahm, T. S. 1996 The dynamics of marginality and self-organized criticality as a paradigm for turbulent transport. Physics of Plasmas 3 (5), 1858-1866.

PARker, J. B. 2015 Zonal flows and turbulence in fluids and plasmas. PhD thesis, Princeton University.

PARker, J. B. 2016 Dynamics of zonal flows: failure of wave-kinetic theory, and new geometrical optics approximations. Journal of Plasma Physics 82 (6), arXiv: 1604.06904.

Pringle, C. C. T., McMillan, B. F. \& Teaca, B. 2017 A nonlinear approach to transition in subcritical plasmas with sheared flow. Physics of Plasmas 24 (12), 122307. 
QIu, Z., Chen, L. \& ZoncA, F. 2018 Kinetic theory of geodesic acoustic modes in toroidal plasmas: a brief review. Plasma Science and Technology 20 (9), 094004.

Rath, F., Peeters, A. G., Buchholz, R., Grosshauser, S. R., Migliano, P., Weikl, A. \& Strintzi, D. 2016 Comparison of gradient and flux driven gyro-kinetic turbulent transport. Physics of Plasmas 23 (5), 052309.

Ruiz, D. E. 2017 A geometric theory of waves and its applications to plasma physics. PhD thesis, Princeton University.

Ruiz, D. E., Guinsky, M. E. \& Dodin, I. Y. 2019 Wave kinetic equation for inhomogeneous drift-wave turbulence beyond the quasilinear approximation. Journal of Plasma Physics $85(1)$.

Ruiz, D. E., Parker, J. B., Shi, E. L. \& Dodin, I. Y. 2016 Zonal-flow dynamics from a phase-space perspective. Physics of Plasmas 23 (12), 122304.

SARAzin, Y. \& GHENDrih, P. 1998 Intermittent particle transport in two-dimensional edge turbulence. Physics of Plasmas 5 (12), 4214-4228.

Sarazin, Y., Grandgirard, V., Abiteboul, J., Allfrey, S., Garbet, X., Ghendrih, P., Latu, G., Strugarek, A. \& Dif-Pradalier, G. 2010 Large scale dynamics in flux driven gyrokinetic turbulence. Nuclear Fusion 50 (5), 054004.

SASAKI, M. 2018 Trapping of turbulence clumps by geodesic acoustic modes. In AIP Conference Proceedings 1993, 020007.

Sasaki, M., Iтоh, K., Kobayashi, T., Kasuya, N., Fujisawa, A. \& Itoh, S.-I. $2018 a$ Propagation direction of geodesic acoustic modes driven by drift wave turbulence. Nuclear Fusion 58 (11), 112005.

Sasaki, M., Kasuya, N., Itoh, K., Hallatschek, K., Lesur, M., Kosuga, Y. \& Itoh, S.-I. 2016 A branch of energetic-particle driven geodesic acoustic modes due to magnetic drift resonance. Physics of Plasmas 23 (10), 102501.

Sasaki, M., Kobayashi, T., Itoh, K., Kasuya, N., Kosuga, Y., Fujisawa, A. \& Itoh, S.-I. $2018 b$ Spatio-temporal dynamics of turbulence trapped in geodesic acoustic modes. Physics of Plasmas 25 (1), 012316.

Schekochinin, A. A., Highcock, E. G. \& Cowley, S. C. 2012 Subcritical fluctuations and suppression of turbulence in differentially rotating gyrokinetic plasmas. Plasma Physics and Controlled Fusion 54 (5), 055011.

Scott, B. \& Smirnov, J. 2010 Energetic consistency and momentum conservation in the gyrokinetic description of tokamak plasmas. Physics of Plasmas 17 (11), 112302.

SRinivasan, K. \& Young, W. R. 2012 Zonostrophic instability. Journal of the Atmospheric Sciences 69 (5), 1633-1656.

Staebler, G. M., Waltz, R. E., Candy, J. \& Kinsey, J. E. 2013 New paradigm for suppression of gyrokinetic turbulence by velocity shear. Phys. Rev. Lett. 110, 055003.

SuchY, K. 1981 Real hamilton equations of geometric optics for media with moderate absorption. Radio Science 16 (6), 1179-1182.

TAYLOR, G. I. 1915 I. Eddy motion in the atmosphere. Philosophical Transactions of the Royal Society of London. Series A, Containing Papers of a Mathematical or Physical Character 215 (523-537), 1-26.

Villard, L., McMillan, B. F., Lanti, E., Ohana, N., Bottino, A., Biancalani, A., Novikau, I., Brunner, S., Sauter, O., Tronko, N. \& Mishchenko, A. 2019 Global turbulence features across marginality and non-local pedestal-core interactions. Plasma Physics and Controlled Fusion 61 (3), 034003.

Waltz, R. E. \& Holland, C. 2008 Numerical experiments on the drift wave-zonal flow paradigm for nonlinear saturation. Physics of Plasmas 15 (12), 122503.

Weinberg, S. 1962 Eikonal method in magnetohydrodynamics. Physical Review 126 (6), 18991909.

Whitham, G. B. 1965 A general approach to linear and non-linear dispersive waves using a Lagrangian. Journal of Fluid Mechanics 22 (2), 273-283.

van Wyk, F., Highcock, E. G., Schekochinin, A. A., Roach, C. M., Field, A. R. \& Dorland, W. 2016 Transition to subcritical turbulence in a tokamak plasma. Journal of Plasma Physics $\mathbf{8 2}$ (6).

Zarzoso, D., Sarazin, Y., Garbet, X., Dumont, R., Strugarek, A., Abiteboul, J., Cartier-Michaud, T., Dif-Pradalier, G., Ghendrih, P., Grandgirard, V., Latu, 
G., Passeron, C. \& Thomine, O. 2013 Impact of energetic-particle-driven geodesic acoustic modes on turbulence. Physical Review Letters 110 (12).

Zhu, H., Zhou, Y. \& Dodin, I. Y. 2018a On the structure of the drifton phase space and its relation to the rayleigh-kuo criterion of the zonal-flow stability. Physics of Plasmas 25 (7), 072121.

Zhu, H., Zhou, Y. \& Dodin, I. Y. 2020 Theory of the Tertiary Instability and the Dimits Shift from Reduced Drift-Wave Models. Physical Review Letters 124 (5).

Zhu, H., Zhou, Y., Ruiz, D. E. \& Dodin, I. Y. $2018 b$ Wave kinetics of drift-wave turbulence and zonal flows beyond the ray approximation. Physical Review E 97 (5), arXiv: $1712.08262 \mathrm{v} 4$. 